\title{
Stochastic recursive optimal control problem with obstacle constraint involving diffusion type control
}

\section{Zhenda $X u^{1 *}$ (i)}

"Correspondence:

zhendaxu@mail.sdu.edu.cn

${ }^{1}$ School of Mathematics, Shandong

University, Jinan, 250100, P.R. China

\section{Springer}

\begin{abstract}
This paper concerns a kind of stochastic optimal control problem with recursive utility described by a reflected backward stochastic differential equation (RBSDE, for short) involving diffusion type control which covers regular control problem, singular control problem and impulse control problem. To begin with, the existence and uniqueness of solution for RBSDEs involving diffusion type control is derived. Then, for the related recursive optimal control problem with obstacle constraint, a sufficient condition to obtain the optimal regular control and diffusion type control is provided. Hence, based on the connection between RBSDE and optimal stopping problem, a class of recursive optimal mixed control problem involving diffusion type control is considered to illustrate our theoretical result, and here the explicit optimal control as well as the stopping time are obtained.
\end{abstract}

MSC: $60 \mathrm{H} 10 ; 49 \mathrm{~N} 25 ; 93 \mathrm{E} 20$

Keywords: Optimal control problem; Reflected backward stochastic differential equation; Diffusion type control; Recursive utility; Sufficient condition

\section{Introduction}

To begin with, El Karoui et al. [1] firstly derived the existence and uniqueness of solutions for backward stochastic differential equations (BSDEs, for short) with obstacle constraint called reflected BSDEs (RBSDEs, for short), which denoted some BSDEs with continuous increasing processes $K$ to keep solutions above the given lower obstacle processes. In addition, Lepeltier and Xu [2] relaxed the continuous obstacles to càglàd obstacles in RBSDEs. To provide the existence and uniqueness result of a solution for the RBSDE with jumps, Hamadène and Ouknine [3] and Crépey and Matoussi [4] kept the local-time-like process $K$ continuous. However, Hamadène and Ouknine [5] let the increasing process $K$ càglàd and used it to follow the negative jumps of obstacles in RBSDE.

RBSDEs have wide applications on mathematical finance and stochastic control. El Karoui, Peng and Quenez [6] formulated stochastic differential recursive utilities introduced by Duffie and Epstein [7] from the perspective of BSDEs. Considering this kind of utility, Wang and Wu [8] obtained the stochastic maximum principle for optimal control

(c) The Author(s) 2020. This article is licensed under a Creative Commons Attribution 4.0 International License, which permits use sharing, adaptation, distribution and reproduction in any medium or format, as long as you give appropriate credit to the original author(s) and the source, provide a link to the Creative Commons licence, and indicate if changes were made. The images or other third party material in this article are included in the article's Creative Commons licence, unless indicated otherwise in a credit line to the material. If material is not included in the article's Creative Commons licence and your intended use is not permitted by statutory regulation or exceeds the permitted use, you will need to obtain permission directly from the copyright holder. To view a copy of this licence, visit http://creativecommons.org/licenses/by/4.0/. 
problems when controllers can only get partial information. Moreover, Zhou [9] obtained sufficient stochastic maximum condition for stochastic recursive optimal control problem. Huang, Wang and $\mathrm{Wu}[10]$ generalized this sufficient condition to the recursive utility which was related to a RBSDE. Moreover, El Karoui, Pardoux and Quenez [11] showed that the pricing problem of an American option can be described by a RBSDE. Applying the results on RBSDEs, Hamadène, Lepeltier and Wu [12] studied differential optimal mixed control problem, where the controller not only can decide a control strategy but also can choose a stopping time to stop the system. On the other hand, for stochastic nonlinear system, Liu, Pan and Cao [13] and Liu et al. [14] proposed the composite learning adaptive dynamic surface control method and adaptive neural network backstepping control method to approximate some uncertain functions.

For optimal control problem involving singular control and impulse control, the stochastic maximum principle was derived by Bahlali and Chala [15] and Dufour and Miller [16], respectively. In addition, using the dynamic programming principle, the optimal control problems involving singular control and impulse control were connected with some quasivariational inequalities by Cadenillas and Zapatero [17] and Haussmann and Suo [18], respectively. Besides, Ma and Yong [19] studied a kind of diffusion type control problem to cover the regular control problem, singular control problem and impulse control problem. Moreover, some kinds of singular control problems were connected with the optimal stopping problems by Dufour and Miller [20], and with the free boundary problems by Dai and Yi [21]. On the other hand, for mathematical finance, Oksendal and Sulem [22] modeled optimal portfolio problems with transaction costs in terms of singular control problems, whereas Cadenillas and Zapatero [17], Ferrari and Vargiolu [23] applied stochastic impulse control to the exchange rate problems. In addition, Wu and Zhang [24] were concerned with a utility maximization problem with the step-shaped consumption strategy by impulse controls.

This paper concerned about some stochastic recursive optimal regular and diffusion type control problems, where the cost functionals were described by some RBSDEs with diffusion type control. The diffusion type control introduced by Ma and Yong [19] is a càglàd process with locally bounded variation paths. By the Lebesgue decomposition, this kind of control processes can be divided into the absolutely continuous part, the singular continuous part and the pure jump part. In addition, the pure jump part could be regard as an impulse control and the others corresponded to singular controls. Different from the optimal control problem studied by Ma and Yong [19], this paper allows for a time variant coefficient of the diffusion type control in state equation and generalizes the cost functional to the solution of a RBSDE involving diffusion type control. Ferrari [25] introduced a kind of stochastic optimal control problem with reflected forward state equation involving singular control and expectation utility. In contrast, we consider non-reflected stochastic state equation involving diffusion type control and recursive utility with obstacle constraint.

Combining the diffusion type control and reflected obstacle constraint, its pure jump part is likely to bring some jumps to the RBSDE. To guarantee the obstacle constraint condition, in general, there are two slightly different approaches to handling some kinds of jumps in RBSDE. The one persists the continuity of local-time-like process $K$, and shares the positive jumps of the Poisson compensated measure term as well as the negative jumps of the càglàd lower obstacle among the proper neighborhoods of $K$ in $[3,4]$. The other 
replaces the continuous process $K$ with a càglàd process to offset a part of those jumps caused by the Poisson compensated measure term in [5], the càglàd obstacle in [2] and the impulse control term for a forward equation in $[25,26]$. Inspired by the above results, we let the càglàd increasing processes $K$ follow the negative jumps of the diffusion type control term, and derive the existence and uniqueness of solution for the RBSDE involving diffusion type control. Hence, unlike [19], we introduce some Hamiltonians with respect to adjoint variables and provide a sufficient condition for a class of stochastic recursive optimal control problem with obstacle constraint involving diffusion type control. This sufficient condition generalizes the result in [10, Theorem 3.2] to diffusion type control problems. Then, similar to the RBSDE case in [27], a corresponding relation between recursive optimal mixed control problems involving diffusion type control and recursive optimal control problems with obstacle constraint involving diffusion type control is proposed. Moreover, to illustrate our results, we consider a class of linear recursive optimal mixed control problem involving diffusion type control, and obtain the optimal stopping time, optimal regular control and optimal diffusion type control with optimal impulse moments.

To explain the motivation of our study and show the application of this kind of optimal control problem, we introduce an example of a recursive utility maximization problem with consumption.

Example 1.1 Suppose there are two kinds of securities in the market. The one is a bond, and the other is a stock described by the following:

$$
\left\{\begin{array} { l } 
{ d S _ { t } ^ { 0 } = r ( t ) S _ { t } ^ { 0 } d t , } \\
{ S _ { s } ^ { 0 } = s _ { 0 } , }
\end{array} \quad \left\{\begin{array}{l}
d S_{t}^{1}=\mu(t) S_{t}^{1} d t+\sigma(t) S_{t}^{1} d W_{t}, \\
S_{s}^{1}=s_{1} .
\end{array}\right.\right.
$$

Here, $W$ is the standard Brownian motion, and $r(\cdot), \sigma(\cdot), \mu(\cdot)$ are deterministic bounded functions with $\mu(\cdot)>r(\cdot)$ and $\sigma(\cdot)^{2}>\delta$. Let $v$ denote the assets invested in the stock and $\eta$ denote the consumption process. Then the wealth process satisfies the following equation:

$$
\left\{\begin{aligned}
d X_{t}^{v, \eta}= & {\left[r(t) X_{t}^{v, \eta}+(\mu(t)-r(t)) v_{t}\right] d t+\sigma(t) v_{t} d W_{t}+G_{t}^{a c} \dot{\eta}_{t}^{a c} d t } \\
& +G_{t}^{s c} d \eta_{t}^{s c}+\theta_{t} d \eta_{t}^{d}, \\
X_{s}^{v, \eta}= & x_{0}
\end{aligned}\right.
$$

where $G^{a c}, G^{s c}, \theta \leq 0$, and $\eta$ is a càglàd process with $\eta_{t}=\eta_{t}^{a c}+\eta_{t}^{s c}+\eta_{t}^{d}, d \eta^{c}=d \eta^{a c}+d \eta^{s c} \geq 0$. This means that the càglàd consumption process covers the classical continuous consumption and the step-shaped consumption in [24].

Based on that, a small investor in this market aims to maximize the recursive utility $J(v, \eta)=Y_{s}^{v, \eta}$ which is always above the obstacle $L_{t} X_{t}^{v, \eta}$ by varying the portfolio strategy $v$ and the consumption strategy $\eta$. The recursive utility is given as follows:

$$
\begin{aligned}
Y_{t}^{v, \eta}= & L_{T} X_{T}^{v, \eta}+\int_{t}^{T}\left[r(m) X_{m}^{v, \eta}-\alpha(m) Y_{m}^{v, \eta}+(\mu(m)-r(m)) v_{m}+F_{m}^{a c} \dot{\eta}_{m}^{a c}\right] d m \\
& +\int_{t}^{T} F_{m}^{s c} d \eta_{m}^{s c}
\end{aligned}
$$




$$
\begin{aligned}
& +\sum_{m \in S_{\eta}[t, T]}\left[\frac{1}{2} S_{m}\left(\Delta \eta_{m}\right)^{2}+\beta_{m} \Delta \eta_{m}\right]+K_{T}^{v, \eta}-K_{t}^{v, \eta}-\int_{t}^{T} Z_{m}^{v, \eta} d W_{m}, \\
& s \leq t \leq T
\end{aligned}
$$

where $\alpha>0, F_{t}^{a c}, F_{t}^{s c} \leq 0$ and $S<0$.

The above maximization problem with consumption can be modeled by a stochastic recursive optimal control problems with obstacle constraint involving diffusion type control.

The rest of this paper is organized as the following. Section 2 formulates the stochastic recursive optimal control problems with obstacle constraint involving diffusion type control, giving the existence and uniqueness of solution for the RBSDEs involving diffusion type control. In Sect. 3, we provide the sufficient condition for optimal control. Hence, to illustrate our theoretical result, a corresponding linear recursive stochastic optimal mixed control problem involving diffusion type control is studied, and the optimal portfolio problem with consumption in Example 1.1 is solved in Sect. 4, where the explicit optimal control and optimal stopping time can be obtained. Section 5 concludes this paper.

\section{Preliminaries and model formulation}

Let $(\Omega, \mathcal{F}, \mathbb{F}, \mathbb{P})$ be a complete filtered probability space with a standard d-dimensional Brownian motion denoted by $W$. Given an initial time $s \geq 0$ and a fixed time horizon $T \geq s$. Moreover, $\mathbb{F}=\left\{\mathcal{F}_{t}^{s}, s \leq t \leq T\right\}$ is a natural filtration with $\mathcal{F}_{t}^{s}=\sigma\left\{W_{r}, s \leq r \leq t\right\}$. Let $|\cdot|$ be the norm and $\langle\cdot, \cdot\rangle$ be the scalar product on a given Euclidean space, and $U, K$ be nonempty convex subset of $\mathbb{R}^{m_{1}}, \mathbb{R}^{m_{2}}$, respectively, with $n, m_{1}, m_{2} \in \mathbb{N}$. In the rest of our paper, we will use the following notation:

$$
\begin{aligned}
& \mathbb{R}_{+}^{m_{2}}=\left\{a \in \mathbb{R}^{m_{2}} \mid a_{i} \geq 0 \text {, where } a_{i} \text { is the ith element of } a, \forall i=1,2, \ldots, m_{2}\right\}, \\
& L_{\mathcal{F}_{T}}^{2}\left(\Omega ; \mathbb{R}^{k}\right)=\left\{\xi: \Omega \rightarrow \mathbb{R}^{k} \mid \xi \text { is } \mathcal{F}_{t}^{s} \text { measurable, } \mathbb{E}|\xi|^{2}<\infty\right\}, \\
& L_{\mathbb{F}}^{2}\left(s, T ; \mathbb{R}^{k}\right)=\left\{\varphi:[s, T] \times \Omega \rightarrow \mathbb{R}^{k} \mid\left\{\varphi_{t}\right\}_{s \leq t \leq T} \text { is } \mathbb{F}\right. \text {-progressively measurable, } \\
& \left.\qquad \mathbb{E}\left[\int_{s}^{T}\left|\varphi_{t}\right|^{2} d t\right]<+\infty\right\}, \\
& S_{\mathbb{F}}^{2}\left(s, T ; \mathbb{R}^{k}\right)=\left\{\varphi:[s, T] \times \Omega \rightarrow \mathbb{R}^{k} \mid\left\{\varphi_{t}\right\}_{s \leq t \leq T} \text { is } \mathbb{F}\right. \text {-adapted, càglàd, } \\
& S_{c i}^{2}=\left\{K:[s, T] \times \Omega \rightarrow[0, \infty) \mid\left\{K_{t}\right\}_{s \leq t \leq T} \text { is } \mathbb{F} \text {-adapted, continuous, increasing, } K_{s}=0,\right. \\
& \left.\quad \mathbb{E}\left[K_{T}^{2}\right]<+\infty\right\}, \\
& S_{\text {cid }}^{2}=\left\{K:[s, T] \times \Omega \rightarrow[0, \infty) \mid\left\{K_{t}\right\}_{s \leq t \leq T} \text { is } \mathbb{F} \text {-adapted, càglàd, increasing, } K_{s}=0,\right. \\
& \left.\quad \mathbb{E}\left[K_{T}^{2}\right]<+\infty\right\}, \\
& \Pi_{c}^{2}=\left\{Z:[s, T] \times \Omega \rightarrow[0, \infty) \mid\left\{Z_{t}\right\}_{s \leq t \leq T} \text { is } \mathbb{F}\right. \text {-supermartingales, continuous, }
\end{aligned}
$$




$$
\begin{gathered}
\left.Z_{T}=0, \text { a.s., } \mathbb{E}\left[\sup _{s \leq t \leq T} Z_{t}^{2}\right]<+\infty\right\}, \\
\mathcal{T}=\{\tau: \Omega \rightarrow[s, T] \mid \tau \text { is } \mathbb{F} \text {-stopping time }\}, \\
S_{\text {sym }}^{n}=\left\{A \mid A \text { is } n \times n \text { matrices, } A^{T}=A\right\} .
\end{gathered}
$$

Any pair $(\nu(\cdot), \eta(\cdot))$ is called an admissible control on $[s, T]$, if $v$, the regular control, belongs to the following Hilbert space:

$$
\begin{aligned}
\mathcal{U}[s, T]= & \{v:[s, T] \times \Omega \rightarrow U \mid v(\cdot) \text { is } \mathbb{F} \text {-progressively measurable, } \\
& \left.\mathbb{E}\left[\int_{s}^{T}|v(t)|^{2} d t\right]<\infty\right\},
\end{aligned}
$$

while $\eta$, the diffusion type control, covering singular control and impulse control, belongs to the following Hilbert space:

$$
\begin{aligned}
\mathcal{K}[s, T]= & \{\varphi:[s, T] \times \Omega \rightarrow K \mid \varphi(\cdot) \text { is càglàd, } \\
& \left.\mathbb{F} \text {-adapted process with bounded variation paths and } \eta_{s}=0\right\} .
\end{aligned}
$$

Similarly to the decomposition of the calculative distribution functions, the càglàd diffusion type control $\eta$ has the following Lebesgue decomposition:

$$
\eta_{t}=\eta_{t}^{a c}+\eta_{t}^{s c}+\eta_{t}^{d}
$$

where $\eta^{a c}$ denotes the absolutely continuous part, $\eta^{s c}$ denotes the singular continuous part, and $\eta^{d}$ denotes the pure jump part of the path $\eta(\omega), \forall \omega \in \Omega$. More precisely, we have $\eta_{t}^{a c}(\cdot)=\int_{s}^{t} \dot{\eta}_{r}^{c}(\cdot) d r$ and $\eta_{t}^{d}(\cdot)=\sum_{s<r \leq t} \Delta \eta_{r}(\cdot)$, where $\Delta \eta_{r}(\cdot)=\eta_{r}(\cdot)-\eta_{r-}(\cdot)$ is a $\mathcal{F}_{r^{-}}$ measurable random variable. Denote $S_{\eta}^{\omega}\left[t_{1}, t_{2}\right]=\left\{r \in\left[t_{1}, t_{2}\right] \mid \Delta \eta_{r}(\omega) \neq 0\right\}$ and $S_{\eta}\left[t_{1}, t_{2}\right]=$ $\left\{(r, \omega) \in\left[t_{1}, t_{2}\right] \times \Omega \mid \Delta \eta_{r}(\omega) \neq 0\right\}, s \leq t_{1} \leq t_{2} \leq T$. To simplify, we use $\sum_{t \in S_{\eta}\left[t_{1}, t_{2}\right]} \xi_{t}$ to represent $\sum_{(t, \omega) \in S_{\eta}\left[t_{1}, t_{2}\right]} \xi_{t}(\omega), \forall(\omega, t, \xi) \in \Omega \times[s, T] \times L_{\mathbb{F}}^{2}\left(s, T ; \mathbb{R}^{k}\right)$. Hence, we assume $\mathbb{P}\{\omega \in$ $\left.\Omega \mid \#\left\{S_{\eta}^{\omega}[s, T]\right\}<\infty\right\}=1$, that is to say, there are finite jumps in the finite interval $[s, T]$ almost surely. Obviously, there is a constant $N>0$ and a sequence $\left\{\sigma_{j}\right\}_{j=1}^{N}$, such that $\sigma_{0} \equiv s$, $\sigma_{1}(\omega)=\inf \left\{t \mid t \in S_{\eta}^{\omega}[s, T]\right\}, \sigma_{j}(\omega)=\inf \left\{t>\sigma_{j-1}(\omega) \mid t \in S_{\eta}^{\omega}[s, T]\right\}, j=2,3, \ldots, N, \inf \{\varnothing\} \triangleq T$, $\mathbb{P}\left\{\omega \in \Omega \mid \sigma_{N-1}(\omega)=T\right\}=1, \sigma_{N} \equiv T$ and $\eta_{t}^{d}=\sum_{j=1}^{N} \Delta \eta_{\sigma_{j}} I_{\left[\sigma_{j}, T\right]}(t)=\sum_{r \in S_{\eta}[s, t]} \Delta \eta_{r}$. Then the sequence of increasing $\mathbb{F}$-stopping times $\left\{\sigma_{j}\right\}$ is called the impulse moment, and the sequence of random variables $\left\{\Delta \eta_{\sigma_{j}}=\eta_{j}\right\}$ is called the impulse magnitude of the diffusion type control $\eta$. Based on that, for some $h^{1}$ and $h^{2}$, we have the following notations:

$$
\begin{aligned}
& \int_{s}^{t} h^{1}(r) d \eta_{r}^{d}=\sum_{j=1}^{N} h^{1}\left(\sigma_{j}\right) \Delta \eta_{\sigma_{j}} I_{\left[\sigma_{j}, t\right]}=\sum_{r \in S_{\eta}[s, t]} h^{1}(r) \Delta \eta_{r}, \\
& \sum_{j=1}^{N} h^{2}\left(\sigma_{j}, \Delta \eta_{\sigma_{j}}\right) I_{\left[t, \sigma_{j}\right]}=\sum_{r \in S_{\eta}[t, T]} h^{2}\left(r, \Delta \eta_{r}\right) .
\end{aligned}
$$

Now we are ready to formulate our system. Consider the following controlled stochastic differential equation (SDE, for short) involving diffusion type control on a finite horizon 
$[s, T]:$

$$
\left\{\begin{aligned}
d x_{t}^{v, \eta} & =b\left(t, x_{t}^{\nu, \eta}, v_{t}\right) d t+\sigma\left(t, x_{t}^{\nu, \eta}, v_{t}\right) d W_{t}+G_{t}^{a c} \dot{\eta}_{t}^{a c} d t+G_{t}^{s c} d \eta_{t}^{s c}+G_{t}^{d} d \eta_{t}^{d} \\
s & \leq t \leq T, \\
x_{s}^{v, \eta} & =\alpha
\end{aligned}\right.
$$

where $x^{v, \eta}$, valued in $\mathbb{R}^{n}$, is the state process, along with $v$, valued in $\mathbb{R}^{m}$, is the regular control process, and $\eta$, valued in $\mathbb{R}$, is the diffusion type control.

Moreover, $\alpha \in \mathbb{R}^{n}$ and the mappings $b(t, x, v):[s, T] \times \mathbb{R}^{n} \times U \rightarrow \mathbb{R}^{n}, \sigma(t, x, v):[s, T] \times$ $\mathbb{R}^{n} \times U \rightarrow \mathbb{R}^{n \times d}$ and $G_{t}^{a c}, G_{t}^{s c}, G_{t}^{d}:[s, T] \rightarrow \mathbb{R}^{n \times m_{2}}$ satisfy the following assumption:

(H2.1) for any $(t, x, v) \in[s, T] \times \mathbb{R}^{n} \times U$, the mappings $b, \sigma$ are continuously differentiable in $x$, the mappings $G_{t}^{a c}, G_{t}^{s c}$ and $G_{t}^{d}$ are continuous and bounded, and there is a constant $\lambda>0$, such that for all $x, x^{\prime} \in \mathbb{R}^{n}$

$$
\begin{aligned}
& |b(t, x, v)| \leq \lambda(1+|x|), \quad|\sigma(t, x, v)| \leq \lambda(1+|x|), \\
& \left|b(t, x, v)-b\left(t, x^{\prime}, v\right)\right|+\left|\sigma(t, x, v)-\sigma\left(t, x^{\prime}, v\right)\right| \leq \lambda\left|x-x^{\prime}\right| .
\end{aligned}
$$

Hence, according to [26, Theorem 4.3], we can easily derive the following result.

Proposition 2.1 Under (H2.1), for any given $\alpha \in \mathbb{R}^{n}$ and $(v, \eta) \in \mathcal{U} \times \mathcal{K}$, the dynamic (2.1) admits a unique solution $x_{t}^{v, \eta} \in S_{\mathbb{F}}^{2}\left(s, T ; \mathbb{R}^{n}\right)$.

Next, we introduce the following controlled RBSDE involving diffusion type control with the lower obstacle $L\left(t, x_{t}^{\nu, \eta}\right) \in S_{\mathbb{F}}^{2}\left(s, T ; \mathbb{R}^{1}\right)$ :

$$
\begin{aligned}
y_{t}^{v, \eta}= & g\left(x_{T}^{\nu, \eta}\right)+\int_{t}^{T} f\left(r, x_{r}^{v, \eta}, y_{r}^{\nu, \eta}, v_{r}\right) d r+K_{T}^{v, \eta}-K_{t}^{\nu, \eta}-\int_{t}^{T} z_{r}^{\nu, \eta} d W_{r} \\
& +\int_{t}^{T} F_{r}^{a c} \dot{\eta}_{r}^{a c} d r+\int_{t}^{T} F_{r}^{s c} d \eta_{r}^{s c}+\sum_{r \in S_{\eta}[t, T]} l\left(r, \Delta \eta_{r}\right), \quad s \leq t \leq T .
\end{aligned}
$$

In addition, the mappings $L(t, x):[s, T] \times \mathbb{R}^{n} \rightarrow \mathbb{R}, g(x): \mathbb{R}^{n} \rightarrow \mathbb{R}, f(t, x, y, v):[s, T] \times \mathbb{R}^{n} \times$ $\mathbb{R} \times U \rightarrow \mathbb{R}, l(t, \xi):[s, T] \times \mathbb{R}^{m_{2}} \rightarrow \mathbb{R}$, and $F_{t}^{a c}, F_{r}^{s c}:[s, T] \rightarrow \mathbb{R}^{1 \times m_{2}}$ satisfy the following assumptions:

(H2.2) for any $(t, x, y, v) \in[s, T] \times \mathbb{R}^{n} \times \mathbb{R} \times U$, the mappings $f, g$ and $L$ are continuously differentiable in $x$ and $y, L(T, x) \leq g(x)$, and there is a constant $\lambda>0$, such that for all $x, x^{\prime} \in \mathbb{R}^{n}, y, y^{\prime} \in \mathbb{R}$,

$$
\begin{aligned}
& |f(t, x, y, v)| \leq \lambda(1+|x|+|y|),|L(t, x)|+|g(x)| \leq \lambda(1+|x|), \\
& \left|f(t, x, y, v)-f\left(t, x^{\prime}, y^{\prime}, v\right)\right|+\left|L(t, x)-L\left(t, x^{\prime}\right)\right|+\left|g(x)-g\left(x^{\prime}\right)\right| \\
& \quad \leq \lambda\left(\left|x-x^{\prime}\right|+\left|y-y^{\prime}\right|\right)
\end{aligned}
$$

(H2.3) $F^{a c}, F^{s c}, l$ are continuous in $t$, and $l$ is continuously differentiable in $\xi$.

Furthermore, in order to define the performance functional, we derive the solvability of the following RBSDE involving diffusion type control (DT-RBSDE, for short) with the 
lower obstacle $L_{t} \in S_{\mathbb{F}}^{2}\left(s, T ; \mathbb{R}^{1}\right)$ :

$$
\begin{aligned}
y_{t}^{\eta}=\zeta & +\int_{t}^{T} h\left(r, y_{r}^{\eta}, z_{r}^{\eta}\right) d r+K_{T}^{\eta}-K_{t}^{\eta}-\int_{t}^{T} z_{r}^{\eta} d W_{r} \\
& +\int_{t}^{T} F_{r}^{a c} \dot{\eta}_{r}^{a c} d r+\int_{t}^{T} F_{r}^{s c} d \eta_{r}^{s c}+\sum_{r \in S_{\eta}[t, T]} l\left(r, \Delta \eta_{r}\right), \quad s \leq t \leq T,
\end{aligned}
$$

where the mapping $h(t, y, z):[s, T] \times \mathbb{R} \times \mathbb{R}^{d} \rightarrow \mathbb{R}$ satisfies the following assumption:

(H2.4) for any $(t, y, z) \in[s, T] \times \mathbb{R} \times \mathbb{R}^{d}$, the mapping $h$ is continuously differentiable in $y$ and $z$, and there exists a constant $\lambda>0$, such that for all $y, y^{\prime} \in \mathbb{R}, z, z^{\prime} \in \mathbb{R}^{d}$,

$$
|h(t, y, z)| \leq \lambda(1+|y|+|z|),\left|h(t, y, z)-h\left(t, y^{\prime}, z^{\prime}\right)\right| \leq \lambda\left(\left|y-y^{\prime}\right|+\left|z-z^{\prime}\right|\right) .
$$

Based on that, we obtain the following solvable result.

Lemma 2.2 Under assumptions (H2.3) and (H2.4), for any given $\eta \in \mathcal{K}$ and $\zeta \in L_{\mathcal{F}_{T}}^{2}(\Omega ; \mathbb{R})$ satisfied $\zeta \geq L_{T}$, (2.3) admits a unique adapted solution $\left(y_{t}^{\eta}, z_{t}^{\eta}, K_{t}^{\eta}\right) \in S_{\mathbb{F}}^{2}\left(s, T ; \mathbb{R}^{1}\right) \times$ $L_{\mathbb{F}}^{2}\left(s, T ; \mathbb{R}^{d}\right) \times S_{\text {cid }}^{2}$, such that

(i) $L_{t} \leq y_{t}^{\eta}$, for all $s \leq t \leq T$;

(ii) $K_{t}^{\eta} \in S_{\text {cid }}^{2}$,

$$
\int_{s}^{T}\left[y_{t}^{\eta}-L_{t}\right] d K_{t}^{\eta}=0
$$

Proof First of all, we consider a special but meaningful case: $l(t, \xi) \equiv 0, \forall(t, \xi) \in[s, T] \times$ $\mathbb{R}^{m_{2}}$.

Let $M_{t}=\int_{t}^{T} F_{r}^{a c} \dot{\eta}_{r}^{a c} d r+\int_{t}^{T} F_{r}^{s c} d \eta_{r}^{s c}, \tilde{h}(t, x, y, v)=h\left(t, x, y+M_{t}, v\right)$ and $\tilde{L}_{t}=L_{t}-M_{t}$. Noting $M_{T}=0$, it is easy to check that $\tilde{L}_{T} \leq g(x), \tilde{h}$ is bounded and Lipschitz in $(y, z)$. Then in virtue of [1, Theorem 5.2], the following RBSDE admits a unique adapted solution $\left(\tilde{y}_{t}^{\eta}, \tilde{z}_{t}^{\eta}, \tilde{K}_{t}^{\eta}\right) \in$ $S_{\mathbb{F}}^{2}\left(s, T ; \mathbb{R}^{1}\right) \times L_{\mathbb{F}}^{2}\left(s, T ; \mathbb{R}^{d}\right) \times S_{c i}^{2}$ :

$$
\left\{\begin{array}{l}
\tilde{y}_{t}^{\eta}=\zeta+\int_{t}^{T} \tilde{h}\left(r, \tilde{y}_{r}^{\eta}, \tilde{z}_{r}^{\eta}\right) d r+\tilde{K}_{T}^{\eta}-\tilde{K}_{t}^{\eta}-\int_{t}^{T} \tilde{z}_{r}^{\eta} d W_{r}, \quad s \leq t \leq T, \\
\tilde{y}_{t}^{\eta} \geq \tilde{L}_{t}, \quad \int_{s}^{T}\left(\tilde{y}_{t}^{\eta}-\tilde{L}_{t}\right) d \tilde{K}_{t}^{\eta}=0 .
\end{array}\right.
$$

Hence, set $y_{t}^{\eta}=\tilde{y}_{t}^{\eta}+M_{t}, z_{t}^{\eta}=\tilde{z}_{t}^{\eta}$ and $K_{t}^{\eta}=\tilde{K}_{t}^{\eta}$, it is easy to check that $\left(y_{t}^{\eta}, z_{t}^{\eta}, K_{t}^{\eta}\right) \in$ $S_{\mathbb{F}}^{2}\left(s, T ; \mathbb{R}^{1}\right) \times L_{\mathbb{F}}^{2}\left(s, T ; \mathbb{R}^{d}\right) \times S_{c i}^{2}$ solves $(2.3)$ with $l(t, \xi) \equiv 0$, and satisfies (2.4) with $L_{t} \leq y_{t}^{\eta}$.

For uniqueness, letting $\left(y^{1}, z^{1}\right)$ and $\left(y^{2}, z^{2}\right)$ be two solutions of $(2.3)$, consider $\left(y^{1}-y^{2}\right)^{2}$. In virtue of Itô's formula and Gronwall's inequality, we derive the uniqueness result.

Next, for the general case, we consider the equivalent equations

$$
\left\{\begin{array}{l}
-d y_{r}^{\eta}=h\left(r, y_{r}^{\eta}, z_{r}^{\eta}\right) d r+d K_{r}^{\eta}-z_{r}^{\eta} d W_{r}+F_{r}^{a c} \dot{\eta}_{r}^{a c} d r+F_{r}^{s c} d \eta_{r}^{s c}, \quad \sigma_{i} \leq r<\sigma_{i+1}, \\
y_{\sigma_{i+1}}^{\eta}=y_{\sigma_{i+1}}^{\eta}+l\left(\sigma_{i+1}, \Delta \eta_{\sigma_{i+1}}\right)+\Delta K_{\sigma_{i+1}}^{\eta}, \quad\left[y_{\sigma_{i+1^{-}}}^{\eta}-L_{\sigma_{i+1}-}\right] \Delta K_{\sigma_{i+1}}^{\eta}=0, \\
y_{r}^{\eta} \geq L_{r}, \quad \int_{\sigma_{i}}^{\sigma_{i+1}}\left(y_{r}^{\eta}-L_{r}\right) d K_{r}^{\eta}=0, \quad y_{T}^{\eta}=\zeta,
\end{array}\right.
$$

where $\Delta K_{t}=K_{t}-K_{t-}, i \geq 0$ and $\sigma_{i} \leq T$,a.s.. To obtain our result, we are sufficient to check the existence and uniqueness of (2.5). For any fixed interval $\left[\sigma_{i}, \sigma_{i+1}\right]$ and any given 
terminal value $\zeta^{i} \in L_{\mathcal{F}_{T}}^{2}(\Omega ; \mathbb{R})$ satisfied $\zeta^{i} \geq L_{\sigma_{i+1}}$, we introduce the following RBSDE for $\left(y^{i}, z^{i}, K^{i}\right)$ :

$$
\left\{\begin{array}{l}
-d y_{r}^{i}=h\left(r, y_{r}^{i}, z_{r}^{i}\right) d r+d K_{r}^{i}-z_{r}^{i} d W_{r}+F_{r}^{a c} \dot{\eta}_{r}^{a c} d r+F_{r}^{s c} d \eta_{r}^{s c}, \quad \sigma_{i} \leq r \leq \sigma_{i+1}, \\
y_{\sigma_{i+1}^{i}}^{i}=\zeta^{i}, \quad y_{r}^{i} \geq L_{r}, \quad \int_{\sigma_{i}}^{\sigma_{i+1}}\left(y_{r}^{i}-L_{r}\right) d K_{r}^{i}=0 .
\end{array}\right.
$$

According to the above discuss, (2.6) admits a unique adapted solution $\left\{\left(y_{r}^{i}, z_{r}^{i}, K_{r}^{i}\right), \sigma_{i} \leq\right.$ $\left.\left.r \leq \sigma_{i+1}\right\} \in S_{\mathbb{F}}^{2}\left(s, T ; \mathbb{R}^{1}\right) \times L_{\mathbb{F}}^{2}\left(s, T ; \mathbb{R}^{d}\right) \times S_{c i}^{2}\right\}$. Noting $y_{T}^{\eta}=\zeta$, by induction from $T$ to $s$, i.e. induction for $i$ from $N$ to 0 , and setting $\zeta^{i}=y_{\sigma_{i+1}}^{\eta}+l\left(\sigma_{i+1}, \Delta \eta_{\sigma_{i+1}}\right)+\Delta K_{\sigma_{i+1}}^{\eta}$, where

$$
\Delta K_{\sigma_{i+1}}^{\eta}=\left(L_{\sigma_{i+1}-}-y_{\sigma_{i+1}}^{\eta}-l\left(\sigma_{i+1}, \Delta \eta_{\sigma_{i+1}}\right)\right)^{+}
$$

we obtain $\left\{\left(y_{r}^{\eta}=y_{r}^{i}, z_{r}^{\eta}=z_{r}^{i}, K_{r}^{i}\right), \sigma_{i} \leq r<\sigma_{i+1}\right\} \in S_{\mathbb{F}}^{2}\left(s, T ; \mathbb{R}^{1}\right) \times L_{\mathbb{F}}^{2}\left(s, T ; \mathbb{R}^{d}\right) \times S_{c i}^{2}$. Then we have the set $K_{t}^{\eta}=\sum_{i \geq 0}\left(K_{\sigma_{i+1}}^{i}+\Delta K_{\sigma_{i+1}}^{\eta}\right) I_{\left\{\sigma_{i+1} \leq t\right\}}+\sum_{i \geq 0} K_{t}^{i} I_{\left\{\sigma_{i}<t<\sigma_{i+1}\right\}}, \forall t \in[s, T]$. We finally get $\left(y_{t}^{\eta}, z_{t}^{\eta}, K_{t}^{\eta}\right) \in S_{\mathbb{F}}^{2}\left(s, T ; \mathbb{R}^{1}\right) \times L_{\mathbb{F}}^{2}\left(s, T ; \mathbb{R}^{d}\right) \times S_{\text {cid }}^{2}$. Hence, we can check that $\left(y_{t}^{\eta}, z_{t}^{\eta}, K_{t}^{\eta}\right)$ solves (2.5) and (2.2). Besides, the uniqueness is obtained at the same time.

\section{Remark 2.3}

(i) If the obstacle $L_{t}$ is continuous, according to the above proof, (2.7) will come to

$$
\Delta K_{\sigma_{i+1}}^{\eta}=\left[-l\left(\sigma_{i+1}, \Delta \eta_{\sigma_{i+1}}\right)\right]^{+} .
$$

That means $K_{t}^{\eta}$ is continuous under $l(t, \xi) \geq 0$ and the jumps of process $K_{t}^{\eta}$ come from the negative jumps of the diffusion type control term. For the reflected forward stochastic differential equation involving impulse control, one can refer to $[25,26]$. Hence, the process $y_{t}^{\eta}$ could be no longer continuous when $l(t, \xi)<0$.

(ii) If the obstacle $L_{t}$ is càglàd, the process $K$ will receive more jumps stemming from the positive jumps of $L_{t}$.

Now, we can obtain the following result and construct our problem.

Theorem 2.4 Under assumptions $(\mathrm{H} 2.1)-(\mathrm{H} 2.3)$, for any given $(v, \eta) \in \mathcal{U} \times \mathcal{K}$ and $\alpha \in$ $\mathbb{R}^{n},(2.1)$ and (2.2) admit a unique adapted solution $\left(x_{t}^{\nu, \eta}, y_{t}^{\nu, \eta}, z_{t}^{\nu, \eta}, K_{t}^{\nu, \eta}\right) \in S_{\mathbb{F}}^{2}\left(s, T ; \mathbb{R}^{n}\right) \times$ $S_{\mathbb{F}}^{2}\left(s, T ; \mathbb{R}^{1}\right) \times L_{\mathbb{F}}^{2}\left(s, T ; \mathbb{R}^{d}\right) \times S_{\text {cid }}^{2}$, such that

(i) $L\left(t, x_{t}^{v, \eta}\right) \leq y_{t}^{v, \eta}, \forall s \leq t \leq T$;

(ii) $K_{t}^{v, \eta} \in S_{\text {cid }}^{2}$,

$$
\int_{s}^{T}\left[y_{t}^{v, \eta}-L\left(t, x_{t}^{v, \eta}\right)\right] d K_{t}^{v, \eta}=0
$$

According to the adaptation of the solution for the controlled DT-RBSDE (2.2), we have $y_{t}^{v, \eta}=\mathbb{E}\left[y_{t}^{v, \eta} \mid \mathcal{F}_{t}^{s}\right]=R_{t}(v, \eta), s \leq t \leq T$ and $J(v, \eta):=y_{s}^{v, \eta}=\mathbb{E}\left[y_{s}^{v, \eta}\right]=R_{s}(\nu, \eta)$, where the performance functional is defined by the following:

$$
R_{t}(v, \eta)=\mathbb{E}\left[g\left(x_{T}^{v, \eta}\right)+\int_{t}^{T} f\left(r, x_{r}^{v, \eta}, y_{r}^{\nu, \eta}, v_{r}\right) d r+K_{T}^{v, \eta}-K_{t}^{v, \eta}\right.
$$




$$
\left.+\int_{t}^{T} F_{r}^{a c} \dot{\eta}_{r}^{a c} d r+\int_{t}^{T} F_{r}^{s c} d \eta_{r}^{s c}+\sum_{r \in S_{\eta}[t, T]} l\left(r, \Delta \eta_{r}\right) \mid \mathcal{F}_{t}^{s}\right], \quad s \leq t \leq T .
$$

When the controller acts with any admissible control strategy $(\nu, \eta)$, the state process and the performance functional are known. Then assume that controller chooses $(v, \eta)$ which is meaningful and generalizes the reward functional $J(v, \eta)=y_{s}^{v, \eta}$. Hence, we can pose the following optimal control problem.

Problem (DT-C): Let assumptions (H2.1)-(H2.3) hold. For the stochastic optimal control problem consisting of the state equation (2.1) and the recursive utility (2.2) of reward functional $J(v, \eta)$, we aim to find an admissible $(\bar{v}, \bar{\eta}) \in \mathcal{U} \times \mathcal{K}$, such that

$$
J(\bar{v}, \bar{\eta})=\sup _{\nu \in \mathcal{U}, \eta \in \mathcal{K}} J(\nu, \eta) \triangleq V(s, x)
$$

where $V(s, x)$ is called value function.

\section{Remark 2.5}

(i) Different from the optimal control problem studied in [19], we generalize the diffusion type control term $\eta_{t}$ to $G_{t}^{a c} \dot{\eta}_{t}^{a c} d t+G_{t}^{s c} d \eta_{t}^{s c}+G_{t}^{d} d \eta_{t}^{d}$ in state equation, and consider a recursive utility with obstacle constraint involving diffusion type control rather than the following expectation utility:

$$
\begin{aligned}
\tilde{J}(v, \eta)= & \mathbb{E}\left\{g\left(x_{T}^{v, \eta}\right)+\int_{s}^{T} f\left(r, x_{r}^{v, \eta}, v_{r}\right) d r+\int_{s}^{T} F_{r}^{a c} \dot{\eta}_{r}^{a c} d r+\int_{s}^{T} F_{r}^{s c} d \eta_{r}^{s c}\right. \\
& \left.+\sum_{r \in S_{\eta}[s, T]} l\left(r, \Delta \eta_{r}\right)\right\} .
\end{aligned}
$$

(ii) Compared with the recursive optimal control problem with obstacle constraint in [10], this paper introduces a diffusion type control in the state equation and the cost functional. We point out that our model covers several kinds of control problem:

(a) the classic regular control problem addressed in [10], when $G_{t}^{a c} \equiv G_{t}^{s c} \equiv G_{t}^{d} \equiv F_{t}^{a c} \equiv F_{t}^{s c} \equiv l(t, \xi) \equiv 0, \forall(t, \xi) \in[s, T] \times \mathbb{R}^{m_{2}}$

(b) the singular control problem, when $b, \sigma$ are independent on $u, l(t, \xi)=F_{t}^{d}|\xi|$ and $F_{t}^{a c} \equiv F_{t}^{s c} \equiv F_{t}^{d}$, $\forall(t, \xi) \in[s, T] \times \mathbb{R}^{m_{2}}$, and without lose any generality, we might as well assume $\dot{\eta}_{t}^{c} \geq 0$

(c) the impulse control problem, when $b, \sigma$ are independent on $u, G_{t}^{a c} \equiv G_{t}^{s c} \equiv F_{t}^{a c} \equiv F_{t}^{s c} \equiv 0, \forall t \in[s, T]$.

\section{Sufficient maximum condition}

In this section, to find the optimal control, we will provide one class of sufficient condition for stochastic recursive optimal control problem with obstacle constraint involving diffusion type control, Problem (DT-C), under the following assumptions:

(H3.1) the partial derivatives of $b, \sigma$ and $f$ in $(x, y)$ are continuous with respect to $(x, y, u, v)$;

(H3.2) $L(t, \cdot)$ is concave for any $t \in[0, T]$.

Hence, the main result in this paper is obtained. 
Theorem 3.1 Let assumptions (H2.1)-(H2.3) and (H3.1)-(H3.2) hold. Assume $(\bar{v}, \bar{\eta})$ is an admissible control, $\bar{x}_{t}$ and $\left(\bar{y}_{t}, \bar{z}_{t}, \bar{K}_{t}\right)$ are the corresponding solutions of (2.1) and (2.2), respectively. For a given $\tau \in \mathcal{T}$, introduce the adjoint processes $P_{t},\left(Q_{t}, q_{t}\right)$ satisfying the following adjoint equations, respectively:

$$
\left\{\begin{array}{l}
d P_{t}=f_{y}\left(t, \bar{x}_{t}, \bar{y}_{t}, \bar{v}_{t}\right) P_{t} d t, \quad s \leq t \leq \tau \\
P_{s}=-1
\end{array}\right.
$$

and

$$
\left\{\begin{aligned}
&-d Q_{t}= {\left[b_{x}^{\top}\left(t, \bar{x}_{t}, \bar{v}_{t}\right) Q_{t}+\sigma_{x}^{\top}\left(t, \bar{x}_{t}, \bar{v}_{t}\right) q_{t}+f_{x}^{\top}\left(t, \bar{x}_{t}, \bar{y}_{t}, \bar{v}_{t}\right) P_{t}\right] d t } \\
&-q_{t} d W_{t}, \quad s \leq t \leq \tau, \\
& Q_{\tau}=\tilde{L}_{x}^{T}\left(\tau, \bar{x}_{\tau}\right) P_{\tau},
\end{aligned}\right.
$$

where $\tilde{L}(t, x)=L(t, x) I_{\{t<T\}}+g(x) I_{\{t=T\}}$. Besides, if for any $\tau \in \mathcal{T}, \mathcal{H}(t, \cdot, \cdot, P, Q, q, \cdot)$ is convex with respect to $x, y, v$, and for any $(t, x, y, P, Q, q, v) \in[0, T] \times \mathbb{R}^{n} \times \mathbb{R} \times \mathbb{R} \times \mathbb{R}^{n} \times \mathbb{R}^{n \times d} \times U$ and $\eta \in \mathcal{K}$,

$$
\begin{aligned}
& \mathcal{H}(t, x, y, P, Q, q, \bar{v}) \leq \mathcal{H}(t, x, y, P, Q, q, v), \\
& \mathbb{E}\left[\int_{s}^{\tau}\left(Q_{t}^{\top} G_{t}^{a c}+P_{t} F_{t}^{a c}\right)\left(\dot{\eta}_{t}^{a c}-\dot{\bar{\eta}}_{t}^{a c}\right) d t+\int_{s}^{\tau}\left(Q_{t}^{\top} G_{t}^{s c}+P_{t} F_{t}^{s c}\right)\left(d \eta_{t}^{s c}-d \bar{\eta}_{t}^{s c}\right)\right. \\
& \left.\quad+\sum_{t \in S_{\bar{\eta}}[s, \tau] \cup S_{\eta}[s, \tau]} Q_{t}^{\top} G_{t}^{d}\left(\Delta \eta_{t}-\Delta \bar{\eta}_{t}\right)+P_{t}\left(l\left(t, \Delta \eta_{t}\right)-l\left(t, \Delta \bar{\eta}_{t}\right)\right)\right] \geq 0,
\end{aligned}
$$

where the Hamiltonian is defined as follows:

$$
\mathcal{H}(t, x, y, P, Q, q, v) \triangleq\langle b(t, x, v), Q\rangle+\langle\sigma(t, x, v), q\rangle+\langle f(t, x, y, v), P\rangle
$$

Then $(\bar{v}, \bar{\eta})$ is optimal of Problem (DT-C).

Proof For any admissible control $(v, \eta), x_{s}^{\nu, \eta}$ is the corresponding state process given by (2.1) and $\left(y_{s}^{v, \eta}, z_{s}^{v, \eta}, A_{s}^{v, \eta}, K_{s}^{v, \eta}\right)$ is the corresponding solution of (2.2). Moreover, recalling that $J(v, \eta)=y_{s}^{\nu, \eta}$, we only need to prove $\bar{y}_{s} \geq y_{s}^{\nu, \eta}$. Define the random variables $\tau$ as follows:

$$
\tau \triangleq \inf \left\{s \leq t \leq T: y_{t}^{v, \eta}=L\left(t, x_{t}^{v, \eta}\right)\right\}
$$

Then $\tau$ is a $\mathcal{F}_{t}^{s}$-stopping times. Hence, $\tau_{0}=s$ and $\tau_{i}=\tau \wedge \sigma_{i}$ also are stopping times. Noting that $P_{s}=-1$, let us consider

$$
y_{s}^{v, \eta}-\bar{y}_{s}=-\mathbb{E}\left[P_{s}\left(y_{s}^{v, \eta}-\bar{y}_{s}\right)\right]
$$

Applying Itô's formula to $P\left(y^{v, \eta}-\bar{y}\right)$ and $\left\langle Q, x^{v, \eta}-\bar{x}\right\rangle$, respectively, on $\left[\tau_{i}, \tau_{i+1}\right)$, then taking expectation and summation, we have

$$
\mathbb{E}\left[P_{\tau}\left(y_{\tau}^{\nu, \eta}-\bar{y}_{\tau}\right)\right]
$$




$$
\begin{aligned}
= & \mathbb{E}\left[P_{s}\left(y_{s}^{v, \eta}-\bar{y}_{s}\right)\right]+\mathbb{E}\left[\int_{s}^{\tau} f_{y}\left(t, \bar{x}_{t}, \bar{y}_{t}, \bar{v}_{t}\right) P_{t}\left(y_{t}^{\nu, \eta}-\bar{y}_{t}\right) d t\right] \\
& -\mathbb{E}\left[\int_{s}^{\tau} P_{t}\left[f\left(t, x_{t}^{v, \eta}, y_{t}^{v, \eta}, v_{t}\right)-f\left(t, \bar{x}_{t}, \bar{y}_{t}, \bar{v}_{t}\right)\right] d t\right] \\
& -\mathbb{E}\left[\int_{s}^{\tau} P_{t} d\left(K_{t}^{v, \eta}-\bar{K}_{t}\right)\right]-\mathbb{E}\left[\int_{s}^{\tau} P_{t} F_{t}^{a c}\left(\dot{\eta}_{t}^{a c}-\dot{\bar{\eta}}_{t}^{a c}\right) d t\right] \\
& -\mathbb{E}\left[\int_{s}^{\tau} P_{t} F_{t}^{s c}\left(d \eta_{t}^{s c}-d \bar{\eta}_{t}^{s c}\right)\right]-\mathbb{E}\left[\sum_{t \in S_{\bar{\eta}}[s, \tau] \cup S_{\eta}[s, \tau]} P_{t}\left(l\left(t, \Delta \eta_{t}\right)-l\left(t, \Delta \bar{\eta}_{t}\right)\right)\right]
\end{aligned}
$$

and

$$
\begin{aligned}
\mathbb{E}\left[\left\langle Q_{\tau}, x_{\tau}^{v, \eta}-\bar{x}_{\tau}\right\rangle\right] & \\
= & \mathbb{E}\left[\left\langle\tilde{L}_{x}^{T}\left(\tau, \bar{x}_{\tau}\right) P_{\tau}, x_{\tau}^{v, \eta}-\bar{x}_{\tau}\right\rangle\right] \\
= & -\mathbb{E}\left[\int_{s}^{\tau}\left\langle b_{x}^{\top}\left(t, \bar{x}_{t}, \bar{v}_{t}\right) Q_{t}+\sigma_{x}^{\top}\left(t, \bar{x}_{t}, \bar{v}_{t}\right) q_{t}+f_{x}^{\top}\left(t, \bar{x}_{t}, \bar{y}_{t}, \bar{v}_{t}\right) P_{t}, x_{t}^{v, \eta}-\bar{x}_{t}\right\rangle d t\right] \\
& +\mathbb{E}\left[\int_{s}^{\tau} Q_{t}^{\top} G_{t}^{a c}\left(\dot{\eta}_{t}^{a c}-\dot{\bar{\eta}}_{t}^{a c}\right) d t+\int_{s}^{\tau} Q_{t}^{\top} G_{t}^{s c}\left(d \eta_{t}^{s c}-d \bar{\eta}_{t}^{s c}\right)+\int_{s}^{\tau} Q_{t}^{\top} G_{t}^{d} d\left(\eta_{t}^{d}-\bar{\eta}_{t}^{d}\right)\right] \\
& +\mathbb{E}\left[\int_{s}^{\tau}\left\langle Q_{t}, b\left(t, x_{t}^{\nu, \eta}, v_{t}\right)-b\left(t, \bar{x}_{t}, \bar{v}_{t}\right)\right\rangle d t\right] \\
& +\mathbb{E}\left[\int_{s}^{\tau}\left\langle q_{t}, \sigma\left(t, x_{t}^{v, \eta}, v_{t}\right)-\sigma\left(t, \bar{x}_{t}, \bar{v}_{t}\right)\right\rangle d t\right] .
\end{aligned}
$$

According to (3.1), the adjoint process $P_{t}$ satisfies $P_{t}<0, t \in[s, \tau]$. Moreover, in virtue of the concavity of obstacle $\tilde{L}(t, \cdot)$, we get

$$
\begin{aligned}
\mathbb{E}\left[P_{\tau}\left(y_{\tau}^{\nu, \eta}-\bar{y}_{\tau}\right)\right] & \geq \mathbb{E}\left[P_{\tau}\left(\tilde{L}\left(\tau, x_{\tau}^{\nu, \eta}\right)-\tilde{L}\left(\tau, \bar{x}_{\tau}\right)\right)\right] \\
& \geq \mathbb{E}\left[P_{\tau}\left(\tilde{L}_{x}^{T}\left(\tau, \bar{x}_{\tau}\right), x_{\tau}^{\nu, \eta}-\bar{x}_{\tau}\right\rangle\right] \\
& =\mathbb{E}\left[\left\langle Q_{\tau}, x_{\tau}^{\nu, \eta}-\bar{x}_{\tau}\right\rangle\right] .
\end{aligned}
$$

Besides, noting that $\bar{K}_{t}$ is increasing and satisfies (2.8), we obtain

$$
\mathbb{E}\left[\int_{s}^{\tau} P_{t}\left(d K_{t}^{\nu, \eta}-d \bar{K}_{t}\right)\right]=-\mathbb{E}\left[\int_{s}^{\tau} P_{t} d \bar{K}_{t}\right] \geq 0
$$

Thus, combining (3.6)-(3.9) and denoting $\Phi_{t}=\left(P_{t}, Q_{t}, q_{t}\right)$, we get

$$
\begin{aligned}
-\mathbb{E}[ & \left.P_{s}\left(y_{s}^{v, \eta}-\bar{y}_{s}\right)\right] \\
\leq & \mathbb{E}\left[\int_{s}^{\tau}\left\langle b_{x}^{T}\left(t, \bar{x}_{t}, \bar{v}_{t}\right) Q_{t}+\sigma_{x}^{T}\left(t, \bar{x}_{t}, \bar{v}_{t}\right) q_{t}+f_{x}^{T}\left(t, \bar{x}_{t}, \bar{y}_{t}, \bar{v}_{t}\right) P_{t}, x_{t}^{v, \eta}-\bar{x}_{t}\right) d t\right] \\
- & \mathbb{E}\left[\int_{s}^{\tau} Q_{t}^{\top} G_{t}^{a c}\left(\dot{\eta}_{t}^{a c}-\dot{\bar{\eta}}_{t}^{a c}\right) d t+\int_{s}^{\tau} Q_{t}^{\top} G_{t}^{s c}\left(d \eta_{t}^{s c}-d \bar{\eta}_{t}^{s c}\right)+\int_{s}^{\tau} Q_{t}^{\top} G_{t}^{d} d\left(\eta_{t}^{d}-\bar{\eta}_{t}^{d}\right)\right] \\
- & \mathbb{E}\left[\int_{s}^{\tau}\left\langle Q_{t}, b\left(t, x_{t}^{v, \eta}, v_{t}\right)-b\left(t, \bar{x}_{t}, \bar{v}_{t}\right)\right\rangle d t\right]-\mathbb{E}\left[\int_{s}^{\tau} P_{t} F_{t}^{a c}\left(\dot{\eta}_{t}^{a c}-\dot{\bar{\eta}}_{t}^{a c}\right) d t\right] \\
- & \mathbb{E}\left[\int_{s}^{\tau} P_{t} F_{t}^{s c}\left(d \eta_{t}^{s c}-d \bar{\eta}_{t}^{s c}\right)\right]-\mathbb{E}\left[\sum_{t \in S_{\bar{\eta}}[s, \tau] \cup S_{\eta}[s, \tau]} P_{t}\left(l\left(t, \Delta \eta_{t}\right)-l\left(t, \Delta \bar{\eta}_{t}\right)\right)\right]
\end{aligned}
$$




$$
\begin{aligned}
& +\mathbb{E}\left[\int_{s}^{\tau} f_{y}\left(t, \bar{x}_{t}, \bar{y}_{t}, \bar{v}_{t}\right) P_{t}\left(y_{t}^{v, \eta}-\bar{y}_{t}\right) d t\right] \\
& -\mathbb{E}\left[\int_{s}^{\tau} P_{t}\left[f\left(t, x_{t}^{v, \eta}, y_{t}^{v, \eta}, v_{t}\right)-f\left(t, \bar{x}_{t}, \bar{y}_{t}, \bar{v}_{t}\right)\right] d t\right] \\
& -\mathbb{E}\left[\int_{s}^{\tau}\left\langle q_{t}, \sigma\left(t, x_{t}^{\nu, \eta}, v_{t}\right)-\sigma\left(t, \bar{x}_{t}, \bar{v}_{t}\right)\right\rangle d t\right] \\
= & I_{1}-I_{2},
\end{aligned}
$$

where

$$
\begin{aligned}
I_{1}= & -\mathbb{E}\left[\int_{s}^{\tau}\left[\mathcal{H}\left(t, x_{t}^{v, \eta}, y_{t}^{\nu, \eta}, \Phi_{t}, v_{t}\right)-\mathcal{H}\left(t, \bar{x}_{t}, \bar{y}_{t}, \Phi_{t}, \overline{\bar{v}}_{t}\right)\right] d t\right] \\
& +\mathbb{E}\left[\int_{s}^{\tau}\left\langle\mathcal{H}_{x}\left(t, \bar{x}_{t}, \bar{y}_{t}, \Phi_{t}, \bar{v}_{t}\right), x_{t}^{\nu, \eta}-\bar{x}_{t}\right) d t\right] \\
& +\mathbb{E}\left[\int_{s}^{\tau} \mathcal{H}_{y}\left(t, \bar{x}_{t}, \bar{y}_{t}, \Phi_{t}, \bar{v}_{t}\right)\left(y_{t}^{\nu, \eta}-\bar{y}_{t}\right) d t\right], \\
I_{2}= & \mathbb{E}\left[\int_{s}^{\tau} Q_{t}^{\top} G_{t}^{a c}\left(\dot{\eta}_{t}^{a c}-\dot{\bar{\eta}}_{t}^{a c}\right) d t+\int_{s}^{\tau} Q_{t}^{\top} G_{t}^{s c}\left(d \eta_{t}^{s c}-d \bar{\eta}_{t}^{s c}\right)\right. \\
& \left.+\int_{s}^{\tau} Q_{t}^{\top} G_{t}^{d} d\left(\eta_{t}^{d}-\bar{\eta}_{t}^{d}\right)\right]+\mathbb{E}\left[\int_{s}^{\tau} P_{t} F_{t}^{a c}\left(\dot{\eta}_{t}^{a c}-\dot{\bar{\eta}}_{t}^{a c}\right) d t\right] \\
& +\mathbb{E}\left[\int_{s}^{\tau} P_{t} F_{t}^{s c}\left(d \eta_{t}^{s c}-d \bar{\eta}_{t}^{s c}\right)\right]+\mathbb{E}\left[\sum_{t \in S_{\bar{\eta}}[s, \tau] \cup S_{\eta}[s, \tau]} P_{t}\left(l\left(t, \Delta \eta_{t}\right)-l\left(t, \Delta \bar{\eta}_{t}\right)\right)\right] \\
\geq & 0 .
\end{aligned}
$$

Next, we prove $I_{1} \leq 0$ to complete our proof. Let $\partial_{u} \varphi(\bar{u})$ denote Clarke's generalized gradient of $\varphi$ with respect to $u$ at $\bar{u} \in G \subseteq \mathbb{R}^{m_{3}}$ given by

$$
\partial_{u} \varphi(\bar{u})=\left\{\xi \in \mathbb{R}^{m_{3}} \mid\langle\xi, b\rangle \leq \limsup _{a \rightarrow \bar{u}, a \in G, \delta \downarrow 0} \frac{\varphi(a+\delta b)-\varphi(a)}{\delta}\right\} .
$$

In fact, in virtue of (3.3), the convexity of $\mathcal{H}$ and [28, Chap. 3], we obtain

$$
\left.\left(\mathcal{H}_{x}, \mathcal{H}_{y}, 0\right)\right|_{\left(t, \bar{x}_{t}, \bar{y}_{t}, \Phi_{t}, \bar{\nu}_{t}\right)} \in \partial_{x, y, v} \mathcal{H}\left(t, \bar{x}_{t}, \bar{y}_{t}, \Phi_{t}, \bar{v}_{t}\right)
$$

Finally, combined with the convexity of Hamiltonian, we obtain

$$
\begin{aligned}
& \mathcal{H}\left(t, x_{t}^{\nu, \eta}, y_{t}^{v, \eta}, \Phi_{t}, v_{t}\right)-\mathcal{H}\left(t, \bar{x}_{t}, \bar{y}_{t}, \Phi_{t}, \bar{v}_{t}\right)-\left\langle\mathcal{H}_{x}\left(t, \bar{x}_{t}, \bar{y}_{t}, \Phi_{t}, \bar{v}_{t}\right), x_{t}^{v, \eta}-\bar{x}_{t}\right\rangle \\
& \quad-\mathcal{H}_{y}\left(t, \bar{x}_{t}, \bar{y}_{t}, \Phi_{t}, \bar{v}_{t}\right)\left(y_{t}^{v, \eta}-\bar{y}_{t}\right) \geq 0,
\end{aligned}
$$

and thus $I_{1}=0$. It implies $J(v, \eta) \leq J(\bar{u}, \bar{v})$.

The proof is completed.

Moreover, we obtain the following result. 


\section{Remark 3.2}

(i) Suppose that those assumptions in Theorem 3.1 hold. In addition, assuming $G_{t}^{a c} \equiv G_{t}^{s c} \equiv F_{t}^{a c} \equiv F_{t}^{s c} \equiv 0$, then Problem (DT-C) comes to an optimal control problem involving impulse control from Remark 2.5-(ii)(c). If for any $\tau \in \mathcal{T}$, $\mathcal{H}(t, \cdot, \cdot, P, Q, q, \cdot)$ is convex in $x, y, v$, and, for any $(t, x, y, P, Q, q, v) \in[0, T] \times \mathbb{R}^{n} \times \mathbb{R} \times \mathbb{R} \times \mathbb{R}^{n} \times \mathbb{R}^{n \times d} \times U$ and $\eta \in \mathcal{K}$,

$$
\begin{aligned}
& \mathcal{H}(t, x, y, P, Q, q, \bar{v}) \leq \mathcal{H}(t, x, y, P, Q, q, v), \\
& \mathbb{E}\left[\sum_{t \in S_{\bar{\eta}}[s, \tau] \cup S_{\eta}[s, \tau]} Q_{t}^{\top} G_{t}^{d}\left(\Delta \eta_{t}-\Delta \bar{\eta}_{t}\right)+P_{t}\left(l\left(t, \Delta \eta_{t}\right)-l\left(t, \Delta \bar{\eta}_{t}\right)\right)\right] \geq 0,
\end{aligned}
$$

then $(\bar{v}, \bar{\eta})$ is optimal of Problem (DT-C).

(ii) Suppose that those assumptions in Theorem 3.1 and in Remark 2.5-(ii)(b) hold. Similarly, Problem (DT-C) leads to an optimal control problem involving singular control. If for any $\tau \in \mathcal{T}, \mathcal{H}(t, \cdot, \cdot, P, Q, q, \cdot)$ is convex in $x, y, v$, and for any $(t, x, y, P, Q, q, v) \in[0, T] \times \mathbb{R}^{n} \times \mathbb{R} \times \mathbb{R} \times \mathbb{R}^{n} \times \mathbb{R}^{n \times d} \times U$,

$$
\begin{aligned}
& \mathcal{H}(t, x, y, P, Q, q, \bar{v}) \leq \mathcal{H}(t, x, y, P, Q, q, v), \\
& \mathbb{E}\left\{\int_{s}^{\tau}\left[Q_{t}^{\top}\left(G_{t}^{a c}+G_{t}^{s c}\right)+P_{t}\left(F_{t}^{a c}+F_{t}^{s c}\right)\right]\left(d \eta_{t}-d \bar{\eta}_{t}\right)\right\} \geq 0,
\end{aligned}
$$

then $(\bar{v}, \bar{\eta})$ is optimal of Problem (DT-C).

(iii) When the impulse domain $K=\mathbb{R}^{m_{2}}$, condition (3.11) can be replaced by

$$
Q_{t}^{\top} G_{t}^{d}\left(\Delta \eta_{t}-\Delta \bar{\eta}_{t}\right)+P_{t}\left(l\left(t, \Delta \eta_{t}\right)-l\left(t, \Delta \bar{\eta}_{t}\right)\right) \geq 0, \quad \forall t \in S_{\bar{\eta}}[s, \tau] \cup S_{\eta}[s, \tau]
$$

\section{Applications to stochastic linear recursive optimal mixed control problems}

This section studies a class of recursive optimal mixed control problem involving diffusion type control, transformed into recursive optimal control problems with obstacle constraint. Hence, applying the result in the above section, we obtain the explicit optimal control and optimal stopping time. Finally, we discuss Example 1.1.

We consider the following linear stochastic system involving regular control and diffusion type control:

$$
\left\{\begin{aligned}
d X_{t}^{v, \eta}= & \left(A_{t} X_{t}^{v, \eta}+B_{t} v_{t}\right) d t+\left(C_{t} X_{t}^{v, \eta}+D_{t} v_{t}\right) d W_{t}+G_{t}^{a c} \dot{\eta}_{t}^{a c} d t+G_{t}^{s c} d \eta_{t}^{s c} \\
& +G_{t}^{d} d \eta_{t}^{d}, \quad s \leq t \leq T \\
X_{s}^{v, \eta}= & x
\end{aligned}\right.
$$

where $X_{t}^{v, \eta}$ is the state process associated with two control processes, including a regular control $v$ and a diffusion type control $\eta=\eta^{a c}+\eta^{s c}+\eta^{d}, x \in \mathbb{R}$, and $A_{t}, B_{t}, C_{t}, D_{t}, G_{t}^{a c}, G_{t}^{s c}$ are $\mathbb{F}$-adapted stochastic process with $G_{t}^{a c}, G_{t}^{s c} \leq 0$.

In the optimal mixed control problem, an intervention action of the controller consists of a control strategy and a stopping time. Denote this action as $(v, \eta ; \varsigma) \in \mathcal{U} \times \mathcal{K} \times \mathcal{T}$. Based on that, state process $X_{t}^{v, \eta}$ keeps controlling until the controller decide to stop it. Then we 
restrict the system to $[s, \varsigma]$, and obtain the following state equation:

$$
\left\{\begin{aligned}
d X_{t}^{v, \eta}= & \left(A_{t} X_{t}^{v, \eta}+B_{t} v_{t}\right) d t+\left(C_{t} X_{t}^{v, \eta}+D_{t} v_{t}\right) d W_{t}+G_{t}^{a c} \dot{\eta}_{t}^{a c} d t \\
& +G_{t}^{s c} d \eta_{t}^{s c}+G_{t}^{d} d \eta_{t}^{d}, \quad s \leq t \leq \varsigma, \\
X_{s}^{v, \eta}= & x .
\end{aligned}\right.
$$

Similar to the stochastic optimal control problem, we introduce the reward functional $\widehat{J}(v, \eta ; \varsigma)=\widehat{R}_{s}(v, \eta ; \varsigma)$ as follows:

$$
\begin{aligned}
\widehat{R}_{t}(v, \eta ; \varsigma)= & \mathbb{E}\left[\int_{t}^{\varsigma \vee t}\left(H_{r} X_{r}^{v, \eta}+N_{r} Y_{r}^{v, \eta}+B_{r} v_{r}+F_{r}^{a c} \dot{\eta}_{r}^{a c}\right) d r\right. \\
& +\int_{t}^{\varsigma \vee t} F_{r}^{s c} d \eta_{r}^{s c}+\sum_{r \in S_{\eta}[t, \varsigma \vee t]} l\left(r, \Delta \eta_{r}\right) \\
& \left.+L_{\zeta \vee t} X_{\zeta \vee t}^{v, \eta} \mid \mathcal{F}_{t}^{s}\right], \quad s \leq t \leq T,
\end{aligned}
$$

where $H_{t}, N_{r}, F_{t}^{a c}, F_{t}^{s c}, l(t, \cdot)$ are $\mathbb{F}$-adapted stochastic process with bounded path, $H_{t} \geq 0$, $F_{t}^{a c}, F_{t}^{s c} \leq 0$, and $L_{t}>0$ is a $\mathbb{R}$-valued deterministic continuous function.

Hence, we pose the following linear stochastic recursive optimal mixed control problem involving diffusion type control:

Problem (DT-LMC): For the state equation (4.2) and the reward functional given by (4.3), we aim to find an admissible control and stopping time $(\bar{v}, \bar{\eta} ; \bar{\zeta}) \in \mathcal{U} \times \mathcal{K} \times \mathcal{T}$, such that

$$
\widehat{J}(v, \eta ; \varsigma) \leq \widehat{J}(\bar{v}, \bar{\eta} ; \bar{\zeta}), \quad \forall(v, \eta ; \varsigma) \in \mathcal{U} \times \mathcal{K} \times \mathcal{T}
$$

Now we concern about a RBSDE which is coupled with (4.1). Compared with (4.3), the lower obstacle could be $L_{t} X_{t}^{v, \eta}$. Hence, this kind of RBSDE can be given as follows:

$$
\begin{aligned}
Y_{t}^{v, \eta}= & L_{T} X_{T}^{v, \eta}+\int_{t}^{T}\left(H_{r} X_{r}^{v, \eta}+N_{r} Y_{r}^{v, \eta}+B_{r} \nu_{r}+F_{r}^{a c} \dot{\eta}_{r}^{a c}\right) d r+\int_{t}^{T} F_{r}^{s c} d \eta_{r}^{s c} \\
& +\sum_{r \in S_{\eta}[t, T]} l\left(r, \Delta \eta_{r}\right)+K_{T}^{\nu, \eta}-K_{t}^{v, \eta}-\int_{t}^{T} Z_{r}^{v, \eta} d W_{r}, \quad s \leq t \leq T .
\end{aligned}
$$

Then, according to Theorem 2.4, (4.4) admits a unique adapted solution denoted by $\left(Y_{t}^{v, \eta}, Z_{t}^{v, \eta}, K_{t}^{v, \eta}\right)$. Moreover, the corresponding reward functional is given by $J(v, \eta)=Y_{s}^{v, \eta}$. Then we can derive a linear stochastic recursive optimal control problem involving diffusion type control with obstacle constraint denoted by Problem (DT-LC) whose objective is to maximize this reward functional over $\mathcal{U} \times \mathcal{K}$ for state equation (4.1) and recursive utility (4.4). Then we show the following connection.

Lemma 4.1 Assume that $(\bar{v}, \bar{\eta})$ is an optimal control of Problem (DT-LC), then $(\bar{v}, \bar{\eta} ; \bar{\zeta})$ is an optimal control and optimal stopping time of Problem (DT-LC), where

$$
\bar{\zeta}=\inf \left\{t \in[s, T]: \bar{Y}_{t}=L_{t} \bar{X}_{t}\right\}
$$


and $\left(\bar{X}_{t}, \bar{Y}_{t}, \bar{Z}_{t}, \bar{K}_{t}\right)$ is the corresponding trajectory of (4.1) and (4.4). We also get the following equality:

$$
V(s, x)=J(\bar{v}, \bar{\eta})=\widehat{J}(\bar{v}, \bar{\eta} ; \bar{\zeta})
$$

Proof Firstly, for any fixed $(\nu, \eta, \varsigma) \in \mathcal{V} \times \mathcal{K} \times \mathcal{T}$ and $t \in[s, T],\left(X_{t}^{v, \eta}, Y_{t}^{v, \eta}, Z_{t}^{v, \eta}, K_{t}^{v, \eta}\right)$ represents the corresponding trajectory. We can obtain the following inequality:

$$
\widehat{R}_{t}(v, \eta ; \varsigma) \leq \widehat{R}_{t}(v, \eta ; \tilde{\varsigma})=Y_{t}^{v, \eta}
$$

where $\tilde{\zeta}=\inf \left\{r \in[t, T]: Y_{r}^{v, \eta}=L_{r} X_{r}^{v, \eta}\right\}$. Actually, noting (4.3), we get

$$
\begin{aligned}
\widehat{R}_{t}(v, \eta ; \varsigma)= & \mathbb{E}\left[\int_{t}^{\varsigma}\left(H_{r} X_{r}^{v, \eta}+N_{r} Y_{r}^{v, \eta}+B_{r} v_{r}+F_{r}^{a c} \dot{\eta}_{r}^{a c}\right) d r\right. \\
& \left.+\int_{t}^{\varsigma} F_{r}^{s c} d \eta_{r}^{s c}+\sum_{r \in S_{\eta}[t, \varsigma]} l\left(r, \Delta \eta_{r}\right)+L_{\varsigma} X_{\varsigma}^{v, \eta} \mid \mathcal{F}_{t}^{s}\right] \\
\leq & \mathbb{E}\left[\int_{t}^{\varsigma}\left(H_{r} X_{r}^{v, \eta}+N_{r} Y_{r}^{v, \eta}+B_{r} v_{r}+F_{r}^{a c} \dot{\eta}_{r}^{a c}\right) d r+\int_{t}^{\varsigma} F_{r}^{s c} d \eta_{r}^{s c}+\sum_{r \in S_{\eta}[t, \varsigma]} l\left(r, \Delta \eta_{r}\right)\right. \\
& \left.+Y_{\varsigma}^{v, \eta}+K_{\varsigma}^{v, \eta}-K_{t}^{v, \eta} \mid \mathcal{F}_{t}^{s}\right] \\
= & Y_{t}^{v, \eta},
\end{aligned}
$$

with $\varsigma=\tilde{\varsigma}$ for equality.

Then, for $(\bar{v}, \bar{\eta})$, we obtain

$$
\widehat{J}(v, \eta ; \varsigma) \leq \widehat{J}(v, \eta ; \tilde{\varsigma})=J(v, \eta) \leq J(\bar{v}, \bar{\eta})=\widehat{J}(\bar{v}, \bar{\eta} ; \bar{\zeta})=V(s, x) .
$$

This lemma illustrates that solving Problem (DT-LC) can be converted into solving Problem (DT-LC) covered by Problem (DT-C).

Let $U=[-1,1], K=\mathbb{R}, d \eta_{t}^{c} \geq 0$, and specially $l\left(t, \Delta \eta_{t}\right)=\frac{1}{2} S_{t}\left(\Delta \eta_{t}\right)^{2}+\beta_{t} \Delta \eta_{t}$, where $S_{t}<$ $0, \beta_{t}$ are $\mathbb{F}$-adapted stochastic process with bounded path. Next, we try to get the explicit optimal control and optimal stopping time of Problem (DT-LC). Firstly, the Hamiltonian is given as follows:

$$
\mathcal{H}(t, x, y, P, Q, q, v)=\left\langle A_{t} x+B_{t} v, Q\right\rangle+\left\langle C_{t} x+D_{t} v, q\right\rangle+\left\langle H_{t} x+N_{t} y+B_{t} v, P\right\rangle .
$$

Hence, according to Theorem 3.1, for arbitrary $\tau \in \mathcal{T}$, the adjoint processes $P_{t}$, and $\left(Q_{t}, q_{t}\right)$ correspond to the following adjoint equation:

$$
\left\{\begin{array}{l}
d P_{t}=N_{t} P_{t} d t, \quad s \leq t \leq \tau, \\
-d Q_{t}=\left(A_{t} Q_{t}+C_{t} q_{t}+H_{t} P_{t}\right) d t-q_{t} d W_{t}, \quad s \leq t \leq \tau, \\
P_{s}=-1, \quad Q_{\tau}=L_{\tau} P_{\tau} .
\end{array}\right.
$$


Recall that $H_{t} \geq 0, F_{t}^{a c} \leq 0, F_{t}^{s c} \leq 0, L_{t}>0$. The explicit expression of adjoint process $Q_{t}$ is obtained as follows: for $t \in[s, T]$,

$$
Q_{t}=\mathbb{E}\left[L_{\tau} P_{\tau} e^{\int_{t}^{\tau}\left(A_{r}-\frac{1}{2} C_{r}^{2}\right) d r+\int_{t}^{\tau} C_{r} d W_{r}}-\int_{t}^{\tau} H_{m} P_{m} e^{\int_{t}^{m}\left(A_{r}-\frac{1}{2} C_{r}^{2}\right) d r+\int_{t}^{m} C_{r} d W_{r}} d m \mid \mathcal{F}_{t}^{s}\right],
$$

where

$$
P_{t}=-e^{\int_{s}^{t} N_{r} d r}
$$

Furthermore, it implies that $P_{t}, Q_{t}<0$. We choose the admissible control as follows:

$$
\bar{v}_{t}=\left\{\begin{array}{ll}
1 & \text { when } B_{t} \geq 0, \\
-1 & \text { when } B_{t}<0,
\end{array} \quad \bar{\eta}_{t}=\sum_{r \in S_{\bar{\eta}}[s, t]}-\frac{Q_{r}^{\top} G_{r}^{d}+P_{r} \beta_{r}}{P_{r} S_{r}},\right.
$$

which means $d \bar{\eta}_{t}^{a c}=d \bar{\eta}_{t}^{s c}=0$. Then (3.3) and (3.4) hold. Therefore (4.9) is an optimal control for Problem (DT-LC). Next, we consider Problem (DT-LC) again with the optimal control (4.9). Recalling the state equation (4.1), we obtain the following equation:

$$
\left\{\begin{array}{l}
d \bar{X}_{t}=\left(A_{t} \bar{X}_{t}+\left|B_{t}\right|\right) d t+\left(C_{t} \bar{X}_{t}+D_{t} \bar{v}_{t}\right) \bar{X}_{t} d W_{t}, \quad \sigma_{i} \leq t \leq \sigma_{i+1}, i=0,1, \ldots, N-1, \\
\bar{X}_{\sigma_{i}}=\bar{X}_{\sigma_{i^{-}}}-G_{\sigma_{i}}^{d} \frac{Q_{\sigma_{i}}^{\top} G_{\sigma_{i}}^{d}+P_{\sigma_{i}} \beta_{\sigma_{i}}}{P_{\sigma_{i}} S_{\sigma_{i}}}, \quad \bar{X}_{s}=x
\end{array}\right.
$$

To solve the above linear SDE with jumps, we present the following result.

Lemma 4.2 Let $\left\{\tau_{i}\right\}_{i=0}^{N}$ be a sequence of $\mathbb{F}$-stopping time, such that $s=\tau_{0} \leq \tau_{1} \leq \cdots \leq \tau_{N}=$ $T$. If the linear $S D E$

$$
\left\{\begin{array}{l}
d x_{t}=\left(a_{t} x_{t}+b_{t}\right) d t+\left(c_{t} x_{t}+d_{t}\right) d W_{t}, \quad \tau_{i} \leq t \leq \tau_{i+1}, i=0,1, \ldots, N-1, \\
x_{\tau_{i}}=x_{\tau_{i-}}+k_{\tau_{i}}, \quad x_{s}=\alpha
\end{array}\right.
$$

admits a unique solution $x_{t} \in S_{\mathbb{F}}^{2}\left(s, T ; \mathbb{R}^{n}\right)$, then

$$
\begin{aligned}
x_{t}= & \alpha e^{\int_{s}^{t}\left(a_{r}-\frac{1}{2}\left|c_{r}\right|^{2}\right) d r+\int_{s}^{t} c_{r} d W_{r}}+\int_{s}^{t} e^{\int_{m}^{t}\left(a_{r}-\frac{1}{2}\left|c_{r}\right|^{2}\right) d r+\int_{m}^{t} c_{r} d W_{r}} b_{m} d m \\
& +\int_{s}^{t} e^{\int_{m}^{t}\left(a_{r}-\frac{1}{2}\left|c_{r}\right|^{2}\right) d r+\int_{m}^{t} c_{r} d W_{r}} d_{m} d W_{m}+\sum_{i=0}^{N} k_{\tau_{i}} I_{\left\{\tau_{i} \leq t\right\}}, \quad s \leq t \leq T .
\end{aligned}
$$

According to the above lemma and (4.1), we obtain

$$
\begin{aligned}
\bar{X}_{t}= & x e^{\int_{s}^{t}\left(A_{r}-\frac{1}{2}\left|C_{r}\right|^{2}\right) d r+\int_{s}^{t} C_{r} d W_{r}}+\int_{s}^{t} e^{\int_{m}^{t}\left(A_{r}-\frac{1}{2}\left|C_{r}\right|^{2}\right) d r+\int_{m}^{t} C_{r} d W_{r}}\left|B_{m}\right| d m \\
& -\sum_{r \in S_{\bar{\eta}}[s, t]} G_{r}^{d} \frac{Q_{r}^{\top} G_{r}^{d}+P_{r} \beta_{r}}{P_{r} S_{r}} \\
& +\int_{s}^{t} e^{\int_{m}^{t}\left(A_{r}-\frac{1}{2}\left|C_{r}\right|^{2}\right) d r+\int_{m}^{t} C_{r} d W_{r}} \frac{D_{m} B_{m}}{\left|B_{m}\right|} d W_{m}, \quad s \leq t \leq T .
\end{aligned}
$$


In virtue of Lemma 4.1, we further obtain the corresponding recursive utility,

$$
\begin{aligned}
\bar{Y}_{t} & =R_{t}(\bar{v}, \bar{\eta})=\widehat{R}_{t}(\bar{v}, \bar{\eta} ; \bar{\zeta}) \\
& =\mathbb{E}\left[\int_{t}^{\bar{\zeta} \vee t}\left[H_{r} \bar{X}_{r}+N_{r} \bar{Y}_{r}+\left|B_{r}\right|\right] d r+\sum_{r \in S_{\bar{\eta}}[t, \bar{\zeta}]} \frac{\left(Q_{r}^{\top} G_{r}^{d}\right)^{2}-\left(P_{r} \beta_{r}\right)^{2}}{2 P_{r} S_{r}}+L_{\bar{\zeta} \vee t} \bar{X}_{\bar{\zeta} \vee t} \mid \mathcal{F}_{t}^{s}\right],
\end{aligned}
$$

the value function,

$$
\begin{aligned}
V(s, x) & =\bar{Y}_{s} \\
& =\mathbb{E}\left[\int_{s}^{\bar{\zeta}}\left[H_{r} \bar{X}_{r}+N_{r} \bar{Y}_{r}+\left|B_{r}\right|\right] d r+\sum_{r \in S_{\bar{\eta}}[s, \bar{\zeta}]} \frac{\left(Q_{r}^{\top} G_{r}^{d}\right)^{2}-\left(P_{r} \beta_{r}\right)^{2}}{2 P_{r} S_{r}}+L_{\bar{\zeta}} \bar{X}_{\bar{\zeta}}\right],
\end{aligned}
$$

the optimal control (4.9) and the optimal stopping time for Problem (DT-LC):

$$
\bar{\zeta}=\inf \left\{t \in[s, T]: \bar{Y}_{t}=L_{t} \bar{X}_{t}\right\} .
$$

Hence, considering $S_{\bar{\eta}}[s, \bar{\zeta}]$, we can further obtain the optimal impulse moment $\left\{\bar{\sigma}_{i}\right\}$ corresponding to the optimal diffusion type control $\bar{\eta}$. We introduce the minimum operator

$$
M[V](s, x)=\sup _{\xi \in K \backslash\{0\}}\left\{V\left(s+, x+G_{s+}^{d} \xi\right)+l(s+, \xi)\right\},
$$

which represents the reward value function with an impulse happening at the very beginning $s$. Noting the $\mathbb{P}\left\{\sigma_{1}>0\right\}>0$ case, we get the following inequality:

$$
M[V](s, x)-V(s, x) \leq 0 .
$$

Meanwhile, combined with the continuity of $Q_{t},(4.9),(4.12)$ and (4.13), we obtain

$$
M[V](s, x)=V\left(s+, x-\frac{Q_{s}^{\top}\left(G_{s}^{d}\right)^{2}+P_{s} \beta_{s} G_{s}^{d}}{P_{s} S_{s}}\right)+\frac{\left(Q_{s}^{\top} G_{s}^{d}\right)^{2}-\left(P_{s} \beta_{s}\right)^{2}}{2 P_{s} S_{s}}
$$

and

$$
\bar{\sigma}_{i+1}=\inf \left\{t \geq \bar{\sigma}_{i}: V\left(t-, \bar{X}_{t-}\right)=M[V]\left(t-, \bar{X}_{t-}\right)\right\}, \quad i=0,1, \ldots, N-1
$$

In the rest of this section, we focus on Example 1.1.

Let $A_{t}=H_{t}=r(t), B_{t}=\mu(t)-r(t)>0, C_{t}=0, D_{t}=\sigma(t), G_{t}^{d}=\theta(t)$ and $N_{t}=-\alpha(t)$. Then the stochastic recursive optimal control problems with obstacle constraint involving diffusion type control can be transformed into this maximization problem with portfolio and consumption. From the above discussion, we obtain the optimal portfolio strategy and consumption strategy as follows:

$$
\begin{aligned}
& \bar{v}_{t}= \begin{cases}1 & \text { when } B_{t} \geq 0, \\
-1 & \text { when } B_{t}<0\end{cases} \\
& \bar{\eta}_{t}=\sum_{r \in S_{\bar{\eta}}[s, t]}-\frac{Q_{r}^{\top} \theta(r)+P_{r} \beta_{r}}{P_{r} S_{r}}=\sum_{i=1}^{N}-\frac{Q_{\bar{\sigma}_{i}}^{\top} \theta\left(\bar{\sigma}_{i}\right)+P_{\bar{\sigma}_{i}} \beta_{\bar{\sigma}_{i}}}{P_{\bar{\sigma}_{i}} S_{\bar{\sigma}_{i}}},
\end{aligned}
$$


and

$$
\bar{\sigma}_{i+1}=\inf \left\{t \geq \bar{\sigma}_{i}: V\left(t-, \bar{X}_{t-}\right)=M[V]\left(t-, \bar{X}_{t-}\right)\right\}, \quad i=0,1, \ldots, N-1
$$

where the adjoint variables satisfy

$$
P_{t}=-e^{\int_{s}^{t}-\alpha(r) d r}, \quad Q_{t}=\mathbb{E}\left[L_{\tau} P_{\tau} e^{\int_{t}^{\tau} r(m) d m}-\int_{t}^{\tau} r(m) P_{m} e^{\int_{t}^{m} r(n) d n} d m \mid \mathcal{F}_{t}^{s}\right],
$$

In addition, the optimal wealth process is given by

$$
\begin{aligned}
\bar{X}_{t}= & x e^{\int_{s}^{t} r(m) d m}+\int_{s}^{t} e^{\int_{m}^{t} r(n) d n}(\mu(m)-r(m)) d m-\sum_{r \in S_{\bar{\eta}}[s, t]} \theta(r) \frac{Q_{r}^{\top} \theta(r)+P_{r} \beta_{r}}{P_{r} S_{r}} \\
& +\int_{s}^{t} e^{\int_{m}^{t} r(n) d n} \sigma(m) d W_{m}, \quad s \leq t \leq T .
\end{aligned}
$$

and the value function satisfies

$$
\begin{aligned}
V(s, x)= & \mathbb{E}\left[\int_{s}^{\bar{\zeta}}\left[r(m) \bar{X}_{m}-\alpha(m) \bar{Y}_{m}+\mu(m)-r(m)\right] d r\right. \\
& \left.+\sum_{r \in S_{\bar{\eta}}[s, \bar{\zeta}]} \frac{\left(Q_{r}^{\top} \theta(r)\right)^{2}-\left(P_{r} \beta_{r}\right)^{2}}{2 P_{r} S_{r}}+L_{\bar{\zeta}} \bar{X}_{\bar{\zeta}}\right] .
\end{aligned}
$$

It is worth pointing out that the random variable $\bar{\zeta}$ denotes the stopping time when the investor prefers to quit the market, to ensure the dynamic minimum recursive utility $L_{t} \bar{X}_{t}$. This stopping time is given by

$$
\bar{\zeta}=\inf \left\{t \in[s, T]: \bar{Y}_{t}=L_{t} \bar{X}_{t}\right\}
$$

Besides, the minimum operator in (4.17) is obtained as follows:

$$
M[V](s, x)=V\left(s+, x-\frac{Q_{s}^{\top}(\theta(s))^{2}+P_{s} \beta_{s} G_{s}^{d}}{P_{s} S_{s}}\right)+\frac{\left(Q_{s}^{\top} \theta(s)\right)^{2}+P_{s} \beta_{s}^{2}}{2 P_{s} S_{s}},
$$

\section{Conclusion}

To the best of our knowledge, it is the first attempt to study a class of stochastic recursive optimal control problem with obstacle constraint involving diffusion type control. There are four distinctive features of our paper. (i) We give the well-posedness of stochastic optimal control problem with obstacle constraint involving diffusion type control. The recursive utility in this problem is given by a RBSDE involving diffusion type control. (ii) We provide a class of sufficient condition to get the stochastic optimal regular control and the optimal diffusion type control. (iii) A kind of optimal portfolio problem with the càglàd consumption strategy is proposed to illustrate our results. (iv) This model covers regular control problem, singular control problem and impulse control problem.

We desire to generalize the control system to the case in which the coefficients related to diffusion type control are allowed to rely on the state process. And we are also still finding more applications including numerical simulations or financial problems. 


\section{Acknowledgements}

The authors would like to thank the editor and the anonymous referees for their helpful suggestions. It is our pleasure to thank Prof. Zhen Wu for kind advice and constructive discussions throughout the work.

\section{Funding}

This work was supported by the National Natural Science Foundation of China (11831010,61961160732), and Natural Science Foundation of Shandong Province (ZR2019ZD42).

\section{Availability of data and materials}

Data sharing is not applicable to this article as no datasets were generated or analysed during the current study.

\section{Competing interests}

The authors declare that they have no competing interests.

\section{Authors' contributions}

The author read and approved the final manuscript.

\section{Publisher's Note}

Springer Nature remains neutral with regard to jurisdictional claims in published maps and institutional affiliations.

\section{Received: 30 January 2020 Accepted: 20 July 2020 Published online: 23 July 2020}

\section{References}

1. El Karoui, N., Kapoudjian, C., Pardoux, É., Peng, S., Quenez, M.-C.: Reflected solutions of backward SDE's, and related obstacle problems for PDE's. Ann. Probab. 25(2), 702-737 (1997)

2. Lepeltier, J.-P., Xu, M.: Penalization method for reflected backward stochastic differential equations with one r.c.I.I. barrier. Stat. Probab. Lett. 75(1), 58-66 (2005)

3. Hamadène, S., Ouknine, Y.: Reflected backward stochastic differential equation with jumps and random obstacle. Electron. J. Probab. 8, 2 (2003)

4. Crépey, S., Matoussi, A.: Reflected and doubly reflected BSDEs with jumps: a priori estimates and comparison. Ann. Appl. Probab. 18(5), 2041-2069 (2008)

5. Hamadène, S., Ouknine, Y.: Reflected backward SDEs with general jumps. Theory Probab. Appl. 60(2), 263-280 (2016)

6. El Karoui, N., Peng, S., Quenez, M.C.: Backward stochastic differential equations in finance. Math. Finance 7(1), 1-71 (1997)

7. Duffie, D., Epstein, L.G.: Stochastic differential utility. Econometrica 60(2), 353-394 (1992)

8. Wang, G., Wu, Z:: The maximum principles for stochastic recursive optimal control problems under partial information. IEEE Trans. Autom. Control 54(6), 1230-1242 (2009)

9. Zhou, X.Y.: Sufficient conditions of optimality for stochastic systems with controllable diffusions. IEEE Trans. Autom. Control 41(8), 1176-1179 (1996)

10. Huang, J., Wang, H., Wu, Z:: A sufficient stochastic maximum principle for a kind of recursive optimal control problem with obstacle constraint. Syst. Control Lett. 114, 27-30 (2018)

11. El Karoui, N., Pardoux, E., Quenez, M.C.: Reflected backward SDEs and American options. In: Rogers, L.C.G., Talay, D. (eds.) Numerical Methods in Finance, vol. 13, pp. 215-231. Cambridge University Press, Cambridge (1997)

12. Hamadène, S., Lepeltier, J.-P., Wu, Z.: Infinite horizon reflected backward stochastic differential equations and applications in mixed control and game problems. Probab. Math. Stat. 19(2), 211-234 (1999)

13. Liu, H., Pan, Y., Cao, J.: Composite learning adaptive dynamic surface control of fractional-order nonlinear systems. IEEE Trans. Cybern. 50(6), 2557-2567 (2019)

14. Liu, H., Pan, Y., Cao, J., Wang, H., Zhou, Y.: Adaptive neural network backstepping control of fractional-order nonlinear systems with actuator faults. IEEE Trans. Neural Netw. Learn. Syst. (2020). https://doi.org/10.1109/TNNLS.2020.2964044

15. Bahlali, S., Chala, A.: The stochastic maximum principle in optimal control of singular diffusions with non linear coefficients. Random Oper. Stoch. Equ. 13(1), 1-10 (2005)

16. Dufour, F., Miller, B.: Necessary conditions for optimal singular stochastic control problems. Stoch. Int. J. Probab. Stoch. Process. 79(5), 469-504 (2007)

17. Cadenillas, A., Zapatero, F:: Classical and impulse stochastic control of the exchange rate using interest rates and reserves. Math. Finance 10(2), 141-156 (2000)

18. Haussmann, U.G., Suo, W.: Singular optimal stochastic controls II: dynamic programming. SIAM J. Control Optim. 33(3), 937-959 (1995)

19. Ma, J., Yong, J.: Dynamic programming for multidimensional stochastic control problems. Acta Math. Sin. 15(4) 485-506 (1999)

20. Dufour, F., Miller, B.: Singular stochastic control problems. SIAM J. Control Optim. 43(2), 708-730 (2004)

21. Dai, M., Yi, F:: Finite-horizon optimal investment with transaction costs: a parabolic double obstacle problem. J. Differ. Equ. 246(4), 1445-1469 (2009)

22. Oksendal, B., Sulem, A.: Optimal consumption and portfolio with both fixed and proportional transaction costs. SIAM J. Control Optim. 40(6), 1765-1790 (2002)

23. Ferrari, G., Vargiolu, T:. On the singular control of exchange rates. Ann. Oper. Res. (2019). https://doi.org/10.1007/s10479-019-03441-6

24. Wu, Z., Zhang, F: Stochastic maximum principle for optimal control problems of forward-backward systems involving impulse controls. IEEE Trans. Autom. Control 56(6), 1401-1406 (2011)

25. Ferrari, G.: On a class of singular stochastic control problems for reflected diffusions. J. Math. Anal. Appl. 473(2), 952-979 (2019) 
26. Ma, J.: Discontinuous reflection, and a class of singular stochastic control problems for diffusions. Stoch. Int. J. Probab. Stoch. Process. 44(3-4), 225-252 (1993)

27. Hamadène, S., Lepeltier, J.-P.: Reflected BSDEs and mixed game problem. Stoch. Process. Appl. 85(2), 177-188 (2000)

28. Yong, J., Zhou, X.Y.: Stochastic Controls: Hamiltonian Systems and HJB Equations, vol. 43. Springer, New York (1999)

Submit your manuscript to a SpringerOpen ${ }^{\circ}$ journal and benefit from:

- Convenient online submission

- Rigorous peer review

- Open access: articles freely available online

- High visibility within the field

- Retaining the copyright to your article

Submit your next manuscript at $\gg$ springeropen.com 